\title{
A Survey on Performance Analysis of Different Multiplexing Scheme used in Passive Optical Network System
}

\author{
Vikas Kumar Mishra \\ M.Tech. Scholar \\ Department of ECE Bhopal \\ Institute of Technology, Bhopal
}

\author{
Bramha S.Tripathi \\ Assistant Professor \\ Department of ECE Bhopal \\ Institute of Technology, Bhopal
}

\author{
Ambikesh P. Gupta \\ Assistant Professor \\ Department of ECE Bhopal \\ Institute of Technology, Bhopal
}

\begin{abstract}
In this research Paper review on various multiplexing techniques used for particularly in optical communication to fulfill the demand of modern technology. Sharing of photonics in development of the physical layer within the future web can most likely increase. The photonics leads currently to a more robust usage of the out there information measure, increase within the transmission rate, increase in energy/power capability measured in W/Gbps, etc. In SCM a single link of transmission medium, there'll be many numbers of transmitters is joined. Every transmitter was tempted to sending information and different types of data simultaneously. We have demonstrated a bidirectional Optical fiber system based on a reflective semiconductor optical amplifier (RSOA) using different modulation technique according to the system. The Optical fiber system was modeled and simulated using commercial software, which is Opti-system 7.0 from Optiwave.
\end{abstract}

\section{Keywords:}

OFDM, OCDMA, SCM, OEO.

\section{INTRODUCTION}

The evolution of electronics and optoelectronic technologies, Microsystems and data technologies, since from three decades, together with a replacement psychological and sociological understanding of the world network, to produce a background for very fast evolutional changes of the Internet [4]. The world network is researched in a very abundant wider context than solely as a complicated technical infrastructure. specific role within the development of the technical, hardware and software system layers play optics, photonics and optoelectronics [5] It looks currently that the photonics, electronics and mechatronics (Microsystems), are going to be ready within the future to make one thing quite solely the advanced technical infrastructure, in a very sort of a totally new reasonably interface of psychological and even physiological nature. It might be a kind of an additional direct interface of human senses to the world network. The mandatory condition is existence of a virtual infrastructure of the throughput none confining the applications, together with quick, period of time transmission of the contents as hi-res 2$\mathrm{D}$ and 3-D pictures. The $100 \mathrm{~GB}$ that is near standardization has recently been commercialized [1]. A next logical step, that is inevitable, is that the one $\mathrm{Tb} / \mathrm{s}$ photonic local area network [2]. This is often associate extension to the quality IEEE 802.3. The backbone network of the longer term net with access links $1 \mathrm{~Tb} / \mathrm{s}$, that is currently researched, goes into the throughputs of one hundred $\mathrm{T}$ bit/s[6]. consecutive step, nowadays still out of reach, may be a network of the throughput reaching four hundred $\mathrm{T}$ bit/s and $1 \mathrm{~Pb} / \mathrm{s}$. nowadays a stable commonplace for one fiber in a very core network, taking into consideration the prices looks ten $\mathrm{T}$ bit/s. The throughput of one $\mathrm{T}$ bit/s looks conjointly even economically and technically, within the shut future, for major local area network access links. Currently the stress is placed on a deeper 'photonization' of the net, even full within the future. This stems from the event of the glass fiber communication and every one optical integrated optics processors. This conjointly stems from the massive prices of producing high speed physics, coming into the domain of $\mathrm{THz}$.

\section{ORTHOGONAL FREQUENCY DIVISION MULTIPLEXING}

OFDM (Orthogonal Frequency Division Multiplexing) is extremely engaging theme for affordable and long reach communication. The output inverse fast Fourier transform after (IFFT) in Associate in Nursing OFDM theme is realvalued, that makes the IQ modulation onto Associate in Nursing RF or optical carrier needless, and thus it reduces the system price. As a sort of multi-carrier modulation technique, OFDM shows high spectrum potency, versatile multi-level committal to writing, and obvious tolerance ISI(Inter SubCarrier Interference) [1]. A high-order coding theme is often used here. Then the serial complex data is split into parallel streams, and every stream is modulated onto $\mathrm{N}$ subcarriers. So as to urge a real-valued time-domain output, a replacement $2 \mathrm{~N}$ points sequence is made, and also the second $\mathrm{N}$ points is that the conjugate sequence of the primary 0.5 . Once $2 \mathrm{~N}$ purpose IFFT, the signaling is of real worth. The cyclic prefix is then inserted for every OFDM image. The generated OFDM signal is had a D/A convertor and afterward used for optical modulation. At the receiver, direct detection is utilized. Once $\mathrm{A} / \mathrm{D}$ conversion, the cyclic prefix is removed and followed by $2 \mathrm{~N}$ points FFT. Solely the primary half the $2 \mathrm{~N}$ points output once FFT is important for signal cryptography. There are some problems in OFDM modulation system. The foremost necessary one is that the high peak-to-average power quantitative relation (PAPR) for multi-carrier modulation. This disadvantage would bring serious distortion within the electrical and optical domains, particularly at the driving power electronic equipment and within the fiber link. It would conjointly cut back the dynamic vary of Associate in Nursing OFDM system [2]. It is documented that OFDM may be a special category of multi-carrier modulation (MCM), a generic implementation of that is delineated in Fig.1. The structure of a complex multiplier factor IQ (modulator/demodulator) that is usually utilized in MCM systems is additionally shown at the lowest of the Fig.1. The key distinction of OFDM from general multicarrier transmission is that the use of orthogonality between the individual subcarriers. 


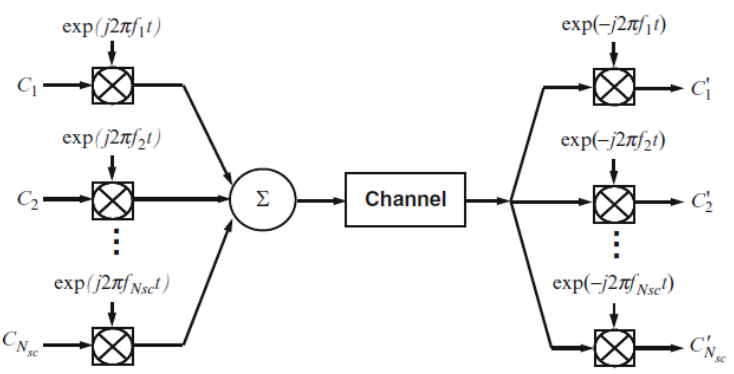

Fig. 1 Conceptual Diagram for a OFDM

Condition for Orthogonality between OFDM Subcarriers and Sub-Bands

The MCM transmitted signal is represented as:

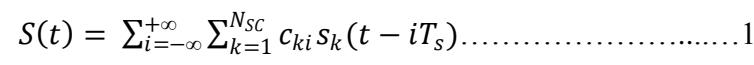

$$
\begin{aligned}
& s_{k}(t)=\prod(t) e^{j 2 \pi f_{k} t} \ldots \ldots \ldots \ldots \ldots \ldots . .2
\end{aligned}
$$

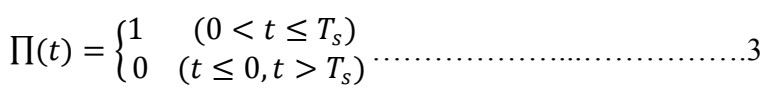

where $c_{k i}$ is the $\mathrm{i}$ th information symbol at the kth subcarrier, $s_{k}$ is the waveform for the kth subcarrier, $N_{S C}$ is the number of subcarriers, $f_{k}$ is the frequency of the subcarrier, and $T_{s}$ is the symbol period, $\Pi(t)$ is the pulse shaping function. A novel approach called OFDM was investigated by employing overlapped yet orthogonal signal set [1-2]. This orthogonality originates from straightforward correlation between any two subcarriers, given by

$$
\begin{aligned}
\delta_{k l}= & \frac{1}{T_{s}} \int_{0}^{T_{s}} s_{k} s^{*} l d t=\frac{1}{T_{s}} \int_{0}^{T_{s}} \exp \left(6 \pi\left(f_{k}-f_{l}\right) t\right) d t \\
= & \exp \left(j \pi\left(f_{k}-f_{l}\right) T_{s}\right) \frac{\sin \left(\pi\left(\left(f_{k}-f_{l}\right) T_{s}\right)\right.}{\pi\left(f_{k}-f_{l}\right) T_{s}}
\end{aligned}
$$

It can be seen that if the following condition

$$
\left(f_{k}-f_{l}\right)=m \frac{1}{T_{s}}
$$

Is satisfied, then the two subcarriers are orthogonal to each other.

\section{SUBCARRIER MULTIPLEXING}

The logical configuration of SCM (Sub Carrier Multiplexing) system is illustrated in figure 2. Much, in single link of transmission medium, there'll be many numbers of transmitters is joined. Every transmitter was tempted to sending information and different sorts of data. So Associate multiplexer for optical electronic device was needed facility the system so as to sharing constant fiber link [8]. As be mentioned above, as a result of its straightforward and lowpriced implementation, high-speed optical information transmission based on SCM technology attracted the eye of the many researchers. The foremost important advantage of SCM in optical communications is its ability to position totally different optical carriers along closely.

Generally, $\mathrm{n}$ numbers of signals were modulated singly with totally different frequency in RF domain. Then the modulated $\mathrm{RF}$ signal are going to be additional up by a RF electronic device (or by an adder) before remodel the RF signal into Optical signal through optical supply and optical modulator on one wavelength. All the operation on top of was perform by one transmitter [9]. Much, in one link of transmission medium, there'll be many numbers of transmitters within the system. Every transmitter was tempted to causation information and different sorts of data. So Associate in multiplexer optical electronic device was needed facility the system so as to sharing constant fiber link. This is as a result of microwave and RF devices square measure rather more mature than optical devices, the soundness of a microwave generator is far higher than Associate in Nursing optical generator (laser diode) and therefore the frequency property of a microwave filter is far higher than Associate in Nursing optical filter. Therefore, the potency of information measure utilization of SCM is anticipated to be far better than typical optical WDM. The most plan of SCM is combining two step of modulation that is working at totally different domain. 1st modulation was occupied at RF half specified many low information measure RF channel carrying analogue or digital signal add up along by mistreatment electronic device.

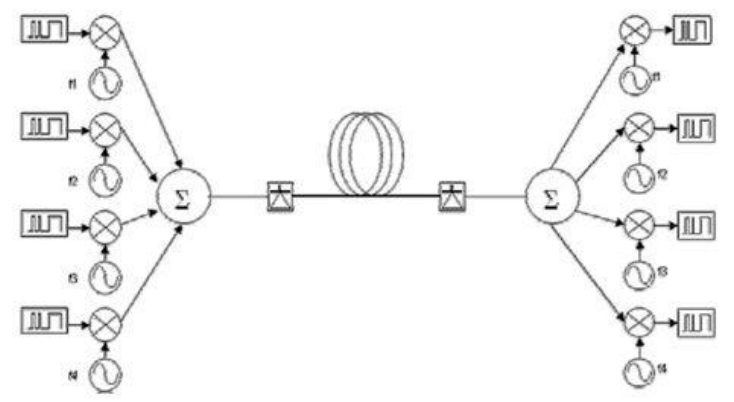

Fig 2 Basic Configuration of SCM

so the signal are going to be terribly near one another within the frequency domain relying to heterodyne oscillator frequency that applied within the modulation half. This combined signal really modulated onto higher frequency microwave carrier. The up-converted signals square measure in numerous frequencies bands and might so be combined by a microwave power combiner forming a microwave subcarrier multiplexed composite signal. Second modulation was occupied at optical domain, the modulated signal then convert to optical domain by mistreatment optical maser diode and optical modulator [10]

\section{OCDMA TECHNIQUE}

From the various multiple access techniques, optical code division multiple access (OCDMA) is obtaining a lot of attraction within the field of all-optical communications as multiple users will access the network asynchronously and at the same time because of the high level of transmission security, large scale bandwidth, and low attenuation and it will thus facilitate several services like prime quality video transmission [3]. OCDMA may be a multiplexing technique by that every line is distinguished by a selected optical code instead of a wavelength or a time slots. At the receiver, the reverse decryption operation is needed to recover the first knowledge. The coding and decryption operation alone represent optical secret writing [4]. Among many varieties of OCDMA systems, spectra amplitude coding (SAC) theme attracts increasing interest as results of multiple access interference (MAI) are often eliminated [5]. In such a system, the optical spectrum is amplitude coded by a special code for every channel to get the OCDMA signals. The incoherent supply seems as a decent candidate for SAC because it is inherently broadband, a necessary characteristic of SAC is shown in fig 3 . 


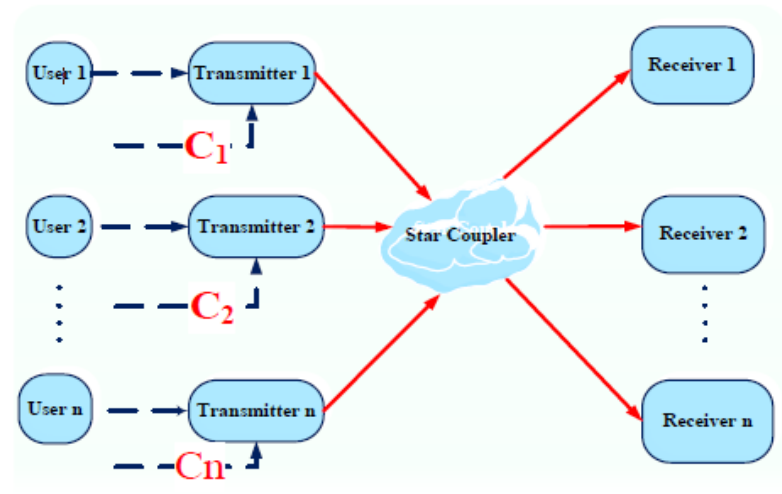

Fig. 3 Optical Code Division Multiple Access System General Structure

\section{PROBLEM FORMULATION}

In the work of previous author they had OFDM and WDM based on DPSK modulation technique whereas in our Paper we have discussed the effective design of Optical system which will be integrated with QPSK type modulation technique and Subcarrier multiplexing (SCM) to model a brand new diagram of a bidirectional transmission Optical system using Reflective Semiconductor Optical Amplifier (RSOA) in downlink and uplink stages. The most idea is to supply an outsized bandwidth to hide the massive data transferred between base stations and also the main office with minimum cost effective system. This may enable the clients to enjoy multimedia services with new updated result in the terms of BER, SNR,Q factor.etc.

\section{PROPOSED WORKS}

On the basis of this review paper we are going to used SCM technique in our farther research works. SCM technique is simple, most efficient and works on larger dynamic range. In this technique temperature \& wave-lengths stabilization are not required and each channel are continuously available and independent for all other channels.

In SAC-OCDMA format, frequency parts of the signal from a broadband optical supply square measure encoded by selection interference or transmittal them in accordance with a signature code. Fig. three shows the principle structure of a SAC-OCDMA system. The receiver filters the incoming signal through a similar direct decoder filter $\mathrm{H}(\mathrm{f})$, that is spectrally clone of a similar encoder at the transmitter, likewise as its complementary decoder $\overline{\mathrm{H}}$ (f), that is that the logical complement of the direct decoder filter $\mathrm{H}(\mathrm{f})$ [6]. The outputs from these decoders square measure detected by two photograph detectors connected in an exceedingly balanced structure. Since the output of the balanced receiver represents the distinction between the two photo detectors' outputs, the meddlesome channels are off whereas the matched channel is demodulated, i.e. MAI is off during this SAC-OCDMA system. Therefore, SAC-OCDMA system was planned as a result of its ability to eliminate the influence of MAI by victimization codes with mounted in section cross-correlation. In recent years, SAC theme of OCDMA has been introduced to eliminate the MAI impact and preserve the orthogonality between users within the system. Many quasi-orthogonal code families square measure utilized in such SAC- OCDMA systems, like maximal-length sequence (M-sequence) codes, Walsh-Hadamard codes, changed quadratic harmony codes, changed double weight so forth. The SAC-OCDMA systems assign one distinctive spectral amplitude code word for every network user to code the amplitude of sunshine supply spectrum. Noises existing within the SAC-OCDMA systems embody shot noise, phase-induced intensity noise (PIIN), and thermal noise, so forth. Solely shot and thermal noise square measure typically thought-about here since PIIN is nearly negligible wherever comparatively low levels of crosscorrelation exist between users [7]. The subsequent analysis is taken from official. [6], provides a simplified plan on the impact of code sequence properties on OCDMA performance. Let $\quad \mathrm{X}=\left(\mathrm{x} \_0, \mathrm{x} \_1, \llbracket \ldots \ldots . \mathrm{x} \rrbracket \_(\mathrm{n}-1)\right) \quad$ and $\mathrm{Y}=\left(\mathrm{y} \_0, \mathrm{y}_{-} 1, \llbracket \ldots . . . \mathrm{y} \rrbracket \_(\mathrm{n}-1)\right)$ be 2 binary sequences such their periodic cross-correlation is

$$
R_{x y}(\tau)=\sum_{i=0}^{n-1} x_{i} y_{i \oplus \tau} \ldots \ldots \ldots \ldots \ldots \ldots \ldots \ldots \ldots \ldots \ldots \ldots
$$

Where " $\oplus$ " denote modulo-n addition

Define the complement of sequence $\mathrm{X}$ to be $\overline{\mathrm{X}}$ $\left.\overline{x_{0}}, \overline{x_{1}} \ldots \ldots \ldots \ldots \overline{x_{n-1}}\right)$, where $\overline{x_{i}}=1-\mathrm{xi}$ The periodic cross correlation sequence between $\overline{\mathrm{X}}$ and $\mathrm{Y}$ is

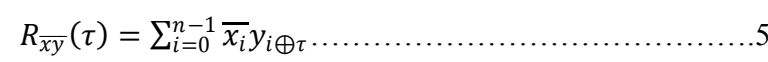

Since $\operatorname{Rxy}(\tau)=\operatorname{R} \bar{x} y(\tau)$, a receiver will reject the interference from a user with the address code $Y$ through computing $\operatorname{Rxy}(\tau)$ - $\operatorname{R} \bar{x} y(\tau)$. Firstly, a unipolar m-sequence $\mathrm{X}=$ $\left(x_{0}, x_{1}, \ldots \ldots . x_{n-1}\right)$ with the period $\mathrm{n}$ is obtained from the bipolar version by replacing each binary 1 by a 1 and each -1 by a 0 . Since the sequence $Y$ is a sequence of cyclical leftsifts of $\mathrm{X}$, i.e., $Y=(T)^{\tau}$ and $X=(X)^{\tau}$, where $\mathrm{T}$ is the operator that shifts vectors cyclically to the left by one place and $\tau$ represents the number of times of cyclic shifts, namely, $\mathrm{TX}=\left(x_{n-1}, x_{0}, x_{1}, x_{n-2}\right)$, its discrete autocorrelation function is

$$
\begin{aligned}
& R_{x \bar{x}}(\tau)=\sum_{i=0}^{n-1} x_{i} x_{i \oplus \tau}= \\
& \left\{\begin{aligned}
\frac{n+1}{2} & \tau \\
\frac{n+1}{4} & 1 \leq \tau \leq n-1
\end{aligned} \ldots \ldots .6\right.
\end{aligned}
$$

These results Obtained from the shift and-add property of an $\mathrm{m}$-sequence, that is, the modulo- 2 addition of an $\mathrm{m}$-sequence and any cyclic shift of the same m-sequence is another cyclic shift of the same sequence. In other words, half the 1 's in $(X)^{\tau}$ coincide with the 1's of $X$ while the other half the 1's in $(X)^{\tau}$ coincide with the 0 's of X.A receiver will reject the signal coming from the interfering subscriber with the address code through computing

$$
\begin{aligned}
& \mathrm{Z}=R_{x y}(\tau)-R_{\bar{x} y}=\sum_{i=0}^{n-1} x_{i} x_{i \oplus \tau}-\sum_{i=0}^{n-1}\left(1-x_{i}\right) x_{i \oplus \tau} \\
& =2 R_{X Y}(\tau)-R_{X Y}(0)=2 \times \frac{n+1}{4}-\frac{n+1}{2}=0 \ldots \ldots \ldots \ldots . \ldots 7
\end{aligned}
$$

Based on this idea, $\mathrm{n}$ cyclic-shift sequences of an m-sequence can be assigned to $\mathrm{n}$ subscribers as their address sequences such that such a network can support $\mathrm{n}$ simultaneous users without any interference, that is, all subscriber address code words can implement completely mutual orthogonality in theory.

\section{PERFORMANCE ANALYSIS}

A comparative analysis is presented in table it's clearly shown that OFDM performance is bitter than other techniques in respect to most of parameter, the table is validated by references taken in the review paper [1-10]. 


\begin{tabular}{|l|l|l|l|}
\hline \multicolumn{1}{|c|}{ Parameter } & OFDM & OCDMA & SCM \\
\hline $\begin{array}{l}\text { Carrier } \\
\text { Frequency }\end{array}$ & THz & THz & Up to GHZ \\
\hline Data rate & $10 \mathrm{Gbit} / \mathrm{s}$ & $622 \mathrm{Mbps}$ & $155 \mathrm{Mbps}$ \\
\hline $\begin{array}{l}\text { Baseband } \\
\text { Bandwidth }\end{array}$ & $25 \mathrm{GHz}$ & $5.6 \mathrm{GHz}$ & $2.5 \mathrm{GHz}$ \\
\hline $\begin{array}{l}\text { Modulation } \\
\text { scheme }\end{array}$ & $\begin{array}{l}\text { QAM,O } \\
\text { OC }\end{array}$ & $\begin{array}{l}\text { OOK,QPS } \\
\text { K }\end{array}$ & $\begin{array}{l}\text { OOK,QPS } \\
\text { K,QAM }\end{array}$ \\
\hline $\begin{array}{l}\text { Total no of } \\
\text { Subcarrier }\end{array}$ & $32-256$ & 80 & 32 \\
\hline $\begin{array}{l}\text { Total no of } \\
\text { Channels }\end{array}$ & 128 & 80 & 32 \\
\hline Bit rate & $1 \mathrm{Tbit} / \mathrm{s}$ & $2.5 \mathrm{Gbit} / \mathrm{s}$ & $4.5 \mathrm{Gbit} / \mathrm{s}$ \\
\hline Fiber Link & $0-1000$ & $\begin{array}{l}60 \text { to } 180 \\
\text { Km }\end{array}$ & $30 \mathrm{Km}$ \\
\hline
\end{tabular}

\section{CONCLUSION}

Optical OFDM transmission has become a quick progressing and vibrant analysis field in fiber communications instead of different one (OCDMA, SCM). Previous couple of years saw experimental demonstrations up to one $\mathrm{Tb} / \mathrm{s}$ transmissions, alongside fast advance in period demonstrations. With the standardization of $100 \mathrm{~Gb}$ and prospect of emergence of the $\mathrm{Tb} / \mathrm{s}$ era, a lot of excitement is growing within the optical communications community for the appliance of OFDM, the modulation format of alternative in RF wireless communications. Within the meanwhile, the analysis in optical OFDM conjointly presents tremendous challenges and opportunities within the areas of novel DSP algorithms, highspeed electronic and photonic integrated circuits etc.

\section{Advantages}

1. One of the main advantages of SCM is that each channel is continuously available and independent of all other channels.

2. There is no need for synchronization between each channel and a high-speed master network clock.

3. Temperature stabilization or wavelength control is not required

\section{REFERENCES}

[1] HAO Yao-hong HUANG Wei-wei HU Wei JIAPeng, Fiber Nonlinearity Mitigation in Long-haul Coherent Optical OFDM,2010 IEEE.
[2] S. L. Jansen, I. Morita, T. C. W. Schenk, D. van den Borne, and H. Tanaka, "Optical OFDM-A candidate for future long-haul optical transmission systems,' presented at the Proc. OFC/NFOEC 2008, San Diego, CA, 2008,Paper, OMU3.

[3] Raad S. Fyath, Howraa M. Mohammad Ali Transmission Performance of Optical Code Division Multiple Access Network Based on Spectral Amplitude Coding,Journal of Emerging Trends in Computing and Information Sciences,VOL. 3, NO. 3, March 2012,pp 444-455.

[4] A. M. Alhassan, N. M. Saad, and N. Badruddin, "An Enhanced Detection Technique for Spectral Amplitude,Coding Optical CDMA Systems", , vol. 23, no. 13, pp. 875-877, 2011, IEEE Photonic Technology Letter.

[5] T. Demeechai, On Noise-limited Performance of Non. complementary Spectral-amplitude coding Optical CDMA Systems,, Vol. 54, Issue 1, 2006, pp. 2931,IEEE Transactions on Communications .

[6] Z. Wei, H. Ghafauri-Shiraz and H. M. H. Shalaby, Performance Analysis of Optical Spectral-amplitudecoding CDMA Systems using a Super-fluorescent Fiber Source, IEEE Photonics Technology Letters, Vol. 13, No.8, 2001, pp. 887-889.

[7] J. H. Wen, J. S. Jhou and C. P. Li, Optical Spectral Amplitude Coding CDMA Systems using Perfect Difference Codes and Interference Estimation", IEE E Proceeding of Optoelectronics, Vol. 153, No. 4, 2006, pp. 152-160.

[8] R. K. Z. Sahbudin, M. K. Abdullah, Performance Analysis of Hybrid SCM/OSCDM System using the Khazani-Syed (KS) Code, WSEAS TRANSACTIONS on COMMUNICATIONS Issue 11, Volume 7 , November2008,pp1066-1074.

[9] P. Laurencio, S. O. Simoes and M. C. R. Medeiros, Impact of the Combined Effect of RIN and Intermodulation Distortion on OSSB/SCM Systems, Journal of LightwaveTechnology, Vol. 24, No. 11, 2006, pp. 4250-4262.

[10] S.Revathi , G.Aarthi, Performance analysis of Wave Length Division and Sub Carrier Multiplexing using different modulation techniques,Vol. 1, Issue 2,pp.317320,IJERA. 